\author{
EVS27 \\ Barcelona, Spain, November 17-20, 2013
}

\title{
Classical EIS and square pattern signals comparison based on a well-known reference impedance
}

\author{
R.AL NAZER ${ }^{1}$, V.CATTIN ${ }^{1}$, P.GRANJON ${ }^{2}$, M.MONTARU ${ }^{3}$, M.RANIERI $^{4}$, V. HEIRIES $^{1}$ \\ ${ }^{1}$ CEA-LETI, Minatec, Grenoble, France \\ ${ }^{2}$ GIPSA-Lab, Saint-Martin-d'Hères, France \\ ${ }^{3}$ INES/CEA, France \\ ${ }^{4}$ CEA-LITEN, Grenoble, France
}

\begin{abstract}
Electrochemical impedance spectroscopy or ac impedance methods are popularly used for the diagnosis of electrochemical generators (batteries or fuel cell). It is now possible to acquire and quantitatively interpret the experimental electrical impedances of such systems, whose evolutions indirectly reflect the modifications of the internal electrochemical process. The scope of these measurement methods is to identify the frequency response function of the system under test by applying a small signal perturbation to the system input, and measuring the corresponding response. Once identified, and according to the application, frequency response functions can provide useful information about the characteristics of the system. Classical EIS consists in applying a set of frequency-controlled sine waves to the input of the system. However, the most difficult problem is the integration of this type of measuring device in embedded systems. In order to overcome this problem, we propose to apply squared pattern excitation signals to perform such impedance measurements. In this paper, we quantify and compare the performance of classical EIS and the proposed broadband identification method applied to a well-known impedance circuit.
\end{abstract}

Keywords: EIS, broadband signals, identification, impedance, Li-ion battery.

\section{Introduction}

Electrochemical Impedance Spectroscopy (EIS) [1] has revealed its importance and value through several applications. It has been shown that it is a powerful device to characterize electrochemical processes occurring in a battery and to thereby estimate useful states indicators [2]. Therefore, it has become a major tool for investigating the properties of batteries in EVs and HEVs [3] [4] [5]. Classical EIS is based on recordings of sine waves injected as an additive perturbation on the input current/voltage signal, and of the corresponding voltage/current response. Though its robustness and high accuracy results, it is still more common in laboratory tests than in online field equipment. [6], [7] and [8] investigate identification methods based on broadband signals. [6] aims to identify biological impedance while [7] and [8] work on battery impedance measurements. These methods have a number of advantages over classical EIS. It requires the application of a short broadband signal [9] [10], and therefore allows the measurement of impedances over a frequency band. 
However, for embedded systems, studies look for simple signals that can be easily generated. In this paper, we focus on squared pattern signals (pseudo random binary sequence or PRBS, square waves) and evaluate their performance relative to those of the classical EIS. This comparison has been performed thanks to a statistical study using a reference passive electric circuit with a well-known impedance.

\section{Spectroscopy}

Classical EIS commonly used in laboratory consists in exciting the system with a small sinusoidal current $i(t)$ of frequency $f$, $i(t)=I_{\max } \sin (2 \pi f t)$, superimposed (or not) to a DC current, and measuring its voltage response $v(t)=V_{\text {max }} \sin (2 \pi f t-\varphi) \quad$ [1]. Therefore, the voltage/current ratio at this particular frequency is expressed as a complex-valued impedance:

$Z_{\text {est }}(f)=\frac{V_{\max }}{I_{\max }} * \exp (-j \varphi)$

Though robustness and accuracy of the results, electrochemical spectroscopy is not suitable for embedded systems like EV and HEV applications due to several reasons. Firstly, an expensive complex electronic is needed to generate sine waves. Secondly, a large frequency band scan with fine frequency resolution takes a long time to be completed. And finally, for embedded systems where the evolution of the impedance must be tracked, the use of the EIS technique imposes a new whole measurement each time an updated impedance estimate is desired. An alternative solution based on broadband identification techniques [11] is proposed in section 3 .

\section{Non parametric identification}

\subsection{Method}

A single input single output (SI/SO) system $\mathcal{H}$ is represented in Fig. 1, where $x[n]$ and $y[n]$ are its input and output signals respectively, and $z[n]$ is its noisy measured output. The unknown measurement noise $b[n]$ is supposed to be additive and uncorrelated with $x[n]$ and therefore with $y[n][12]$.

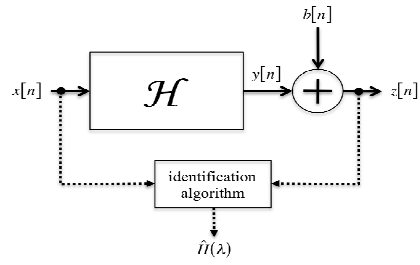

Fig. 1. Non-parametric identification of a (SI/SO) LTI system $\mathcal{H}$ in the frequency domain.

If $\mathcal{H}$ is linear and time invariant (LTI), it is completely characterized by its frequency response function $H(\lambda)$, where $\left.\lambda \in\rfloor-\frac{1}{2}, \frac{1}{2}\right\rfloor$ is the normalized frequency, leading to the frequency $f$ in Hertz when multiplied by the sampling frequency. Indeed, for periodic deterministic and stationary random signals, the frequency domain input-output relationship of such systems is simply:

$$
S_{z x}(\lambda)=S_{y x}(\lambda)=H(\lambda) S_{x x}(\lambda)
$$

where $S_{x x}(\lambda)$ is the power spectral density (PSD) of $x[n]$ and $S_{y x}(\lambda)$ is the cross power spectral density (CPSD) between $x[n]$ and $y[n]$. Eq. (2) is the foundation of non-parametric identification of LTI systems in the frequency domain [13] [14]. Therefore, on the frequency bands where the input PSD verifies $S_{x x}(\lambda) \neq 0$, the unknown frequency response function $H(\lambda)$ can be calculated through:

$$
H(\lambda)=\frac{S_{z x}(\lambda)}{S_{x x}(\lambda)} \text { if } S_{x x}(\lambda) \neq 0 .
$$

This finally leads to a frequency domain identification method of the unknown system $\mathcal{H}$. Eq. (3) shows that PSDs and CPSDs must be estimated in order to estimate the desired frequency response function $H(\lambda)$, which is realized by using Welch modified periodogram [15]. Measured signals are first split-up into $L$ data segments of length $N$. All these segments are then windowed by a window function $w[n]$ of length $N$, and the discrete Fourier transform of each windowed segment is computed by the use of the fast Fourier transform algorithm. Finally, products of these discrete Fourier transforms are averaged in order to estimate the desired spectral quantities. As an example, the corresponding 
estimator of the CPSD between $z[n]$ and $x[n]$ is given by:

$$
\hat{S}_{z x}(\lambda)=\frac{A}{L} \sum_{k=0}^{L-1} Z_{k}(\lambda) X_{k}^{*}(\lambda)
$$

where:

- $\quad A$ is a normalization factor,

- $Z_{k}(\lambda)$ (resp. $X_{k}(\lambda)$ ) is the discrete Fourier transform of the $k^{\text {th }}$ windowed segment of $z[n]$ (resp. $x[n])$,

- $\quad{ }^{*}$ denotes the complex conjugate.

Similarly, the estimator of the PSD of $x[n]$ $\hat{S}_{x x}(\lambda)$ is obtained by replacing $Z_{k}(\lambda)$ by $X_{k}(\lambda)$ in Eq. (4).

Simple estimators can now be obtained to estimate the desired frequency response function $H(\lambda)$ by using Eq. (4) in Eq. (3) leading to:

$\hat{H}(\lambda)=\frac{\hat{S}_{z x}(\lambda)}{\hat{S}_{x x}(\lambda)}$ if $\hat{S}_{x x}(\lambda) \neq 0$

Finally, Eq. (4) and (5) constitute the "identification algorithm" appearing in Fig. 1 that is used to estimate the frequency response function $H(\lambda)$ of an unknown LTI system through its input $x[n]$ and noisy measured output $z[n]$.

\subsection{Square pattern signals}

In order to estimate the whole electrical impedance, the input signal should be able to excite the system in the frequency band of interest.

We choose two particular signals: the pseudo random binary sequence (PRBS) which is a broadband signal since it presents an almost flat power spectrum, and a square version of EIS where square waves are applied instead of sine waves. These particular signals have been chosen since they are based on squared patterns, and are therefore easily implemented in embedded systems.

\subsubsection{Pseudo random binary sequence}

A PRBS is a deterministic periodic sequence of length $N$ bits that switches between two levels $+\mathrm{A}$ and $-\mathrm{A}$. And by choosing the time for one bit $T_{b i t}$ corresponding to the bit frequency
$F_{b i t}=\frac{1}{T_{b i t}}$, the highest frequency that will be excited is considered to be $f_{\text {max }}=0.4^{*} F_{b i t}$ as a good rule of thumb, while the lowest one is $f_{\min }=F_{b i t} / \mathrm{N}$. The corresponding PRBS then presents an almost flat power spectrum over the frequency

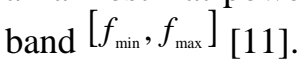

\subsubsection{Square EIS version}

A square version of the classical EIS is also developed. A square wave of frequency $f$ is used where $f$ scans the frequency band of interest. This signal is a suitable signal for embedded applications since a square wave can be simply generated by the analog drive of a transistor.

\section{Experimental considerations}

\subsection{Square pattern signal generation}

An electronic circuit was designed especially to perform the experiments on an electrochemical source (like a battery). Only three main devices allow the generation of input currents with square patterns (square version EIS and PRBS). A transistor is controlled by a microcontroller where the desired signal is implemented and a few other basic components (like a small current driver to better drive the transistor's gate). A resistance $\mathrm{R}$ is inserted in series with the transistor to control the amplitude of the input current (given that the nominal voltage of the system under test does not change during the test). By controlling the transistor switch on or off, a desired amount of current delivered by the electrochemical source is derived in the resistance $\mathrm{R}$. Therefore, the current seen by the system to identify follows the desired squared pattern. This circuit, described in Fig. 2, has to be connected carefully to the system to identify. It respects the four-terminal measurement technique [1]. It is extremely important when measuring low impedance devices such as batteries, fuel cells and ultra-capacitors. When measuring very low impedance cells, the impedance of the connection cables may be of the same order of magnitude as the impedance of the cell itself. The 4-terminal technique ensures that the voltage drop measured by the instrument is measured directly across the cell and does not include the voltage drop in the cables, and therefore provides accurate measurement of the cell voltage. 


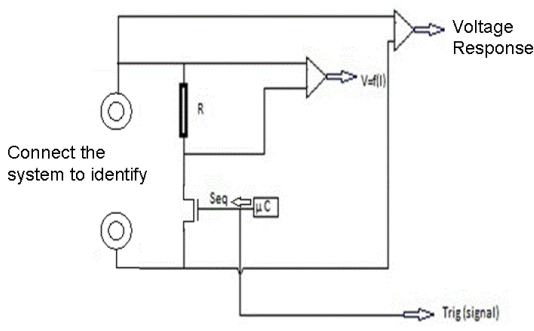

Fig. 2. Simplified structure of the electronic used to generate square pattern currents.

The acquisition of the input current (calculated from the voltage drop measured across the resistor R) and the corresponding output voltage response has been performed under a large SNR level thanks to an OR-36, a high performance acquisition device (24 bits).

\subsection{Classical EIS}

The classical EIS has been performed with a VMP-300 Biologic system, using a booster of 10A. EC-Lab® software, supplied with the VMP-300, is used to select spectroscopy parameters (frequencies, number of periods, sine amplitude...).

\subsection{Input signals characteristics}

In what follows, the classical EIS, its square version and the previous non-parametric identification method using a PRBS are performed on a well-known impedance circuit. The identified frequency response functions obtained by these different methods are then compared to the theoretical one. As an example, we focus on the frequency band from 4 to $96 \mathrm{~Hz}$. All the signals have the same total time duration of approximately 5 seconds.

For PRBS, a sequence of $N=63$ bits with frequency bit $F_{b i t}=240 \mathrm{~Hz}$ is considered. It leads to frequency band limits $f_{\min }=F_{b i t} / N \approx 4 \mathrm{~Hz}$ and $f_{\max }=0.4 * F_{b i t}=0.4 * 240=96 \mathrm{~Hz}$. Data are split-up into $\mathrm{L}=18$ disjoint segments of length $N_{w}=2688$ samples. Consequently, each segment has a time duration of $T=N_{w} / f_{s}=0.2625$ seconds, which results in a frequency resolution of $1 / T=4 \mathrm{~Hz}$.

For classical and square version EIS, the fundamental frequency scans the set of frequencies between $4 \mathrm{~Hz}$ and $96 \mathrm{~Hz}$ with a frequency step of $4 \mathrm{~Hz}$, and 5 fundamental periods are used for each measurement step.

\subsection{Reference circuit}

In order to compare EIS and the broadband method, a passive electrical circuit with a wellknown impedance is used (Fig. 3(a)). It consists of a resistance $\left(R_{1}=200 \mathrm{~m} \Omega \pm 1 \%\right)$ in series with a parallel bridge of a resistance $\left(R_{2}=1 \Omega \pm 1 \%\right)$ and a capacitor $\left(C_{2}=820 \mu F \pm 1 \%\right)$. The Nyquist diagram of such a circuit is a portion of a circle in the frequency band of interest with a "resonant" frequency equal to $194 \mathrm{~Hz}$. A parasitic resistance $R_{2 p}=12 \mathrm{~m} \Omega \pm 1 \%$ is given by the manufacturer and is considered to be in series with $C_{2}$.

\subsection{Experimental protocol}

As the electronic of Fig. 2 is designed with passive components, it requires the use of an external energy source (like a battery) and a two-step measurement protocol (Fig. 3(b)). A Li-ion battery is connected at J4. In this work, we used a graphite/LiFePO4 cell with a nominal capacity of 2.3 Ah (ANR26650m1 battery from A123 Systems Company Ltd). At J6 is connected either the VMP-300 biologic system or the electronic described in Fig. 2.

A set of jumpers $(\mathrm{JPi}, \mathrm{i}=1, . ., 4)$ is used to switch between the two steps of the measurement protocol. Firstly, if JP1 and JP3 are "on", the current is applied only to the battery cell and its voltage response is measured. This set of data leads to identify only the battery impedance $\hat{Z}_{1}(\lambda)$. Secondly, the activation of JP2 and JP4 provides the set of data to identify impedance $\hat{Z}_{2}(\lambda)$, consisting of the sum of the battery impedance and the passive reference circuit impedance.

The impedance of the passive reference circuit alone can then be estimated by:

$$
\hat{Z}_{\text {passive_circuit }}(\lambda)=\hat{Z}_{2}(\lambda)-\hat{Z}_{1}(\lambda)
$$

To perform this experiment, it is important to keep in mind that the battery must behave as a linear and time invariant system. This is verified if the two following conditions are satisfied:

- the additive input current is sufficiently low in magnitude,

- the battery is in a stationary state, or its impedance is stable for the duration of the measurement, and this corresponds to a SOC between $60 \%$ and $80 \%$ for the used $\mathrm{Li}$-ion battery over the selected frequency band $(4 \mathrm{~Hz}, 96 \mathrm{~Hz})$. 


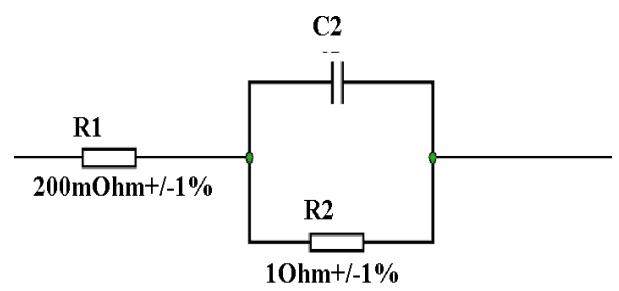

(b)

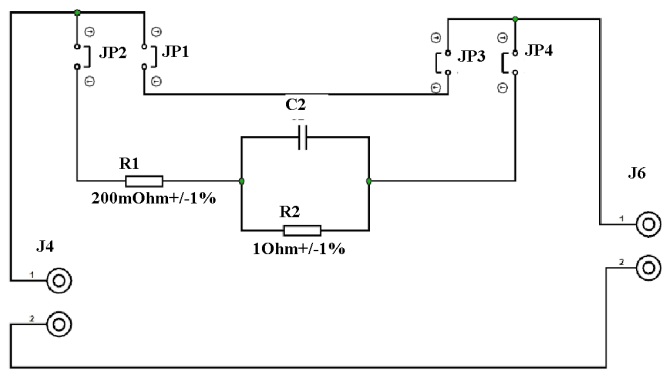

Fig. 3. Passive reference circuit structure.

\section{Experimental results}

\subsection{Estimation error quantification}

The analytical expression of the impedance of the reference circuit can be computed from the electrical equivalent circuit given in Fig. 3(a). It is then compared with the estimated electrical impedances in order to evaluate the quality of the three identification methods through a statistical study. For each input signal, 30 impedance measurements of the passive reference circuit are done. This leads to 60 sets of measurements (alternate Z1 and Z2 impedances) from which we compute the normalized root mean square estimation error NRMSE in \% defined by Eq. (7). This error quantifies the averaged normalized difference between the estimated impedance $\hat{Z}$ and its theoretical value $Z$ averaged over realizations and over the selected frequency band for a specific excitation signal. The normalized standard deviation Nstd in \% is also given in Eq.(7) in order to characterize the error dispersion:

$$
\begin{aligned}
& \left.\operatorname{MSE}(f)=\operatorname{mean}_{k}|\hat{Z}(k, f)-Z(f)|^{2}\right] \\
& \operatorname{Variance}(f)=\operatorname{mean}_{k}\left[\mid \hat{Z}(k, f)-\operatorname{mean}_{k}[\hat{Z}(f)]^{2}\right] \\
& N R M S E_{\%}=100 * \sqrt{\frac{\operatorname{mean}_{f}[\operatorname{mSE}(f)]}{\operatorname{mean}_{f}\left[\left.Z(f)\right|^{2}\right]}} \\
& \operatorname{NStd}_{\%}=100 * \sqrt{\frac{\operatorname{mean}_{f}[\operatorname{Variance}(f)]}{\operatorname{mean}_{f}\left[\left.Z(f)\right|^{2}\right]}}
\end{aligned}
$$

where $\hat{Z}(k, f)$ is the electrical impedance obtained at frequency $f$ with the $k^{\text {th }}$ realization.

\subsection{Classical EIS results}

In Fig. , the Nyquist plot of the theoretical impedance of the passive reference circuit is represented in blue. It is known within an interval due to the accuracy on the components values given by the manufacturer. The same impedance obtained by averaging 30 impedances estimated by a classical EIS is also plotted in green in the same figure. For this experiment, a NRMSE of $0.69 \%$ and a Nstd of $0.41 \%$ are obtained.

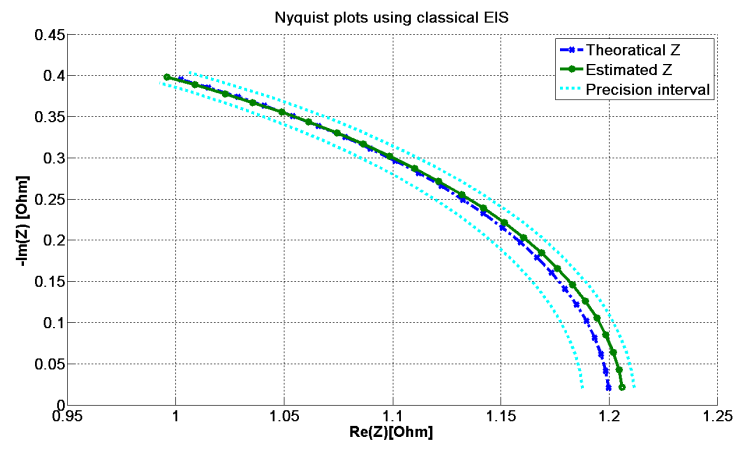

Fig. 4. Nyquist plot results using an EIS laboratory apparatus.

\subsection{Square version EIS results}

A set of 30 estimations of the impedance of the passive reference circuit is also obtained with a square EIS approach. The corresponding results are plotted in Fig. 5. A NRMSE of $2.44 \%$ and a Nstd of $0.14 \%$ are obtained. 


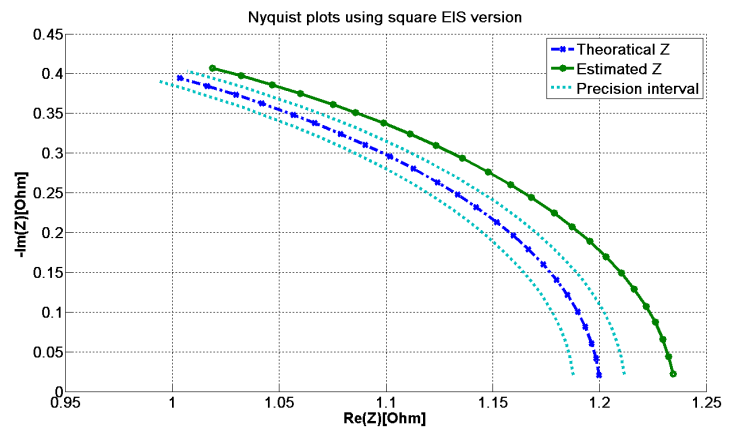

Fig. 5. Nyquist plot results using a square EIS version.

\subsection{PRBS results}

The PRBS described in 3.2.2 is used to estimate the same impedance in the same frequency band. The results are plotted in Fig. 6, where a NRMSE of $2.6 \%$ and a Nstd of $0.19 \%$ are observed.

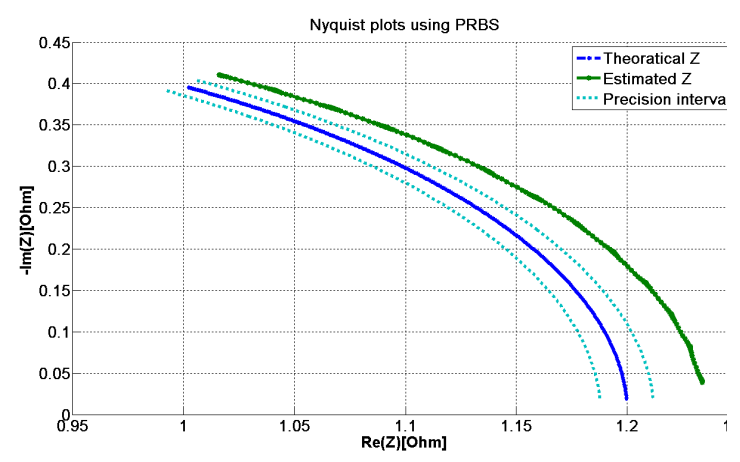

Fig. 6. Nyquist plot results using a PRBS.

\subsection{Discussion}

We infer from these results that impedances can be identified with signals constituted of square patterns (PRBS and square waves) with an estimation error which has a low dispersion (similar value of the Nstd parameter for the three methods), but a bias that cannot be neglected. The NRMSE which include the bias information is quite the same for the two methods including square patterns (squared EIS and PRBS). Several reasons may explain this difference with classical EIS. Firstly, connectors on the circuit vary slightly between classical EIS and square pattern signals methods. Secondly, in the experimental protocol we can only use a discharge current, so that the mean dc part of the additive input current is not zero and affects the voltage measurements. However, the low dispersion of the estimation error reveals that square pattern signals could be useful for specific embedded applications. For example, in the field of the EVs and HEVs where the battery impedance is desired in order to estimate state of charge and state of health quantities, we need an accurate relative estimation. Based on a first impedance measurement at an initial time, we can track the temporal evolution of this quantity with an excellent relative accuracy.

\section{Conclusion}

This paper focuses on the usefulness of squared pattern excitation signals for the identification of system impedance. A non-parametric identification method has been theoretically introduced. Experimental results ascertain that signals based on square pattern like square waves and PRBS lead to a biased broadband identification, but with a very low dispersion. Consequently, such signals are suitable for applications based on relative impedance tracking, like state of charge or state of health monitoring.

\section{References}

[1] Barsoukov, E. and Macdonald, J. R. (eds) (2005). Impedance Spectroscopy: Theory, Experiment, and Applications, Second Edition, John Wiley \& Sons, Inc., Hoboken, NJ, USA.

[2] K.S.Champlin, US Patent 6,037,777,(2000)

[3] F. Huet, "A review of impedance measurements for determination of the state-of-charge or state-ofhealth of secondary batteries," Journal of Power Sources, vol. 70, pp. 59-69, 1998.

[4] Buller S., (2002). Impedance-Based Simulation Models for Energy Storage Devices in Advanced Automotive Power Systems, Thesis, Institute for power electronics and electrical drives ISEA, Aachen/Germany.

[5] ORAZEM M. E., TRIBOLLET B., (2008), Electrochemical impedance spectroscopy, John Wiley \& Sons, Inc., Hoboken, NJ, USA.

[6] Shady GAWAd, Tao Sun, Nicolas G. Green and Hywel Morgan (2007), Impedance spectroscopy using maximum length sequences: Application to single cell analysis, Review of Scientific Instruments, Volume 78.

[7] Al Nazer R., Cattin V., Granjon P. and al, (2013), Broadband Identification of Battery Electrical Impedance for HEV, IEEE Transactions on vehicular technology, Volume 62, no. 7

[8] Faireweather A.J., Foster M.P., Stone D.A., Battery parameter identification with Pseudo Random Binary Sequence excitation (PRBS), (2011), Journal of Power Sources, Volume 196, pp. 9398-9406.

[9] S.C. Creason, J.W. Hayes, D.E. Smith (1973), Fourier transform faradic admittance measurements II. Comparison of measurement 
efficiency for various test signal waveforms, Journal of Electroanalytical chemistry and interfacial electrochemistry, Volume 47, p.9-46

[10] C. Gabrielli (1992). Comparison of sine wave and white noise analysis for electrochemical impedance measurements, Electroanalytical Chemistry, Volume 335, p.33-53.

[11] Pintelon R., Schoukens J., (2001). System identification, a frequency domain approach. First Edition, IEEE press marketing, Piscataway,NJ (ISBN 0-7803-6000-1)

[12] Bendat J.S., Piersol A.G. (2010). Random data: analysis and measurements procedures. Fourth Edition, John Wiley \& Sons, Inc., Hoboken, NJ, USA

[13] Jenkins G.M. and Watts D.G., (1968). Spectral analysis and its applications. Holden day, San Francisco, USA

[14] Shin K., Hammond J.K., (Ed) (2008). Fundamentals of Signal Processing for Sound and Vibration Engineers. West Sussex PO19 8SQ, England

[15] Brillinger D. R. (2001). Time Series: Data Analysis and Theory, SIAM San Fransisco,USA.

\section{Authors}

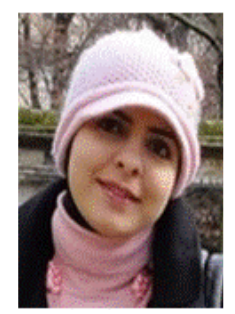

R. AL NAZER received an engineering degree in electronics from Lebanese University-Faculty of Engineering, Lebanon. Then, she got a research master dispensation from the "Doctoral college of Grenoble Institute of Technology" and is currently pursuing the PHD degree at the CEALETI (Electronics and Information Technology Laboratory). Her recent interest is focused on impedance identification of the battery implemented in hybrid electric and electric vehicles.

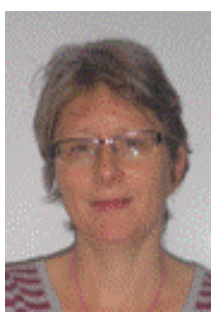

V. CATTIN After an engineering degree in electronics and signal processing and a $\mathrm{PhD}$ in signal image and speech processing, I work at the CEA since 1998 as a research engineer in the fields of detection and localisation from magnetometers and accelerometers, as a leader of industrial and collaborative projects on motion capture and drift measurement of cars. I supervised two PhDs and several students on signal processing, recently applied to battery diagnosis and monitoring. I am currently in charge of a laboratory working on advanced electronics for energy and power applications

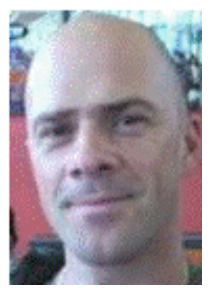

P. GRANJON received its PhD. degree from the Grenoble Institute of Technology (INPG), France in 2000. He joined the Laboratoire des Images et des Signaux (LIS) in 2002 and the Gipsa-lab at INPG in 2007, where he holds a position as associate professor. His current research is mainly focused on signal processing applications in diagnosis and electrical engineering, such as fault diagnosis in electrical systems, power networks, rotating machinery and batteries

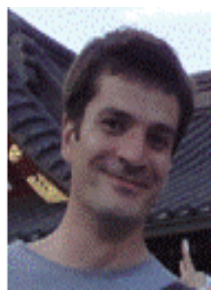

M. MONTARU received the Ph.D. degree from the Grenoble Institute of Technology (INPG), France in 2009. $\mathrm{He}$ joined the Electrical Storage Laboratory (LSE), where he holds a position as researcher engineer. His current research is mainly focused on battery behavior and ageing modeling in order to enhance battery states indicators and management.

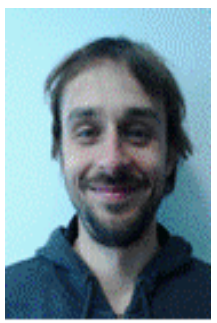

M. RANIERI was born in Ravenna (Italy) on the 20th of April 1979. He graduated in Electronic Engineering with Bio-Medical specialisation at the "Università degli Studi di Bologna" (Italy), inside the "Facoltà di Ingegneria" department, in 2005. As a member of Cea research center, he first worked on current and voltage sensors' benchmarking for automotive applications. Nowadays he researches on BMS (Battery Management System) for Lithium-ion based electric vehicles and other portable devices. 